\section{Methodological Issues in Studying Sex-specific Relationships of Serum Uric Acid with All-cause Mortality in Adults with Normal Kidney Function}

\section{To the Editor:}

We have read the paper by Kang and colleagues that was published in The Journal of Rheumatology in March 2017 with great interest ${ }^{1}$. The authors proposed to examine the clinical effect of serum uric acid (SUA) levels as a risk factor for mortality, considering exclusion of kidney function. The authors concluded that the SUA-mortality relationship differed by sex, so that lower SUA was independently associated with higher risk of all-cause mortality in men with normal kidney function. Although the present study makes a valuable contribution to the area, some methodological points should be considered.

First, the authors used the Cox proportional hazard $(\mathrm{PH})$ regression model to examine the association between the SUA and all-cause mortality, which is problematic. The main assumption for this regression model is $\mathrm{PH}$ and its results are not valid when the $\mathrm{PH}$ assumption is violated. As reported in their Figure 3, the HR is absolutely different across the SUA values, and this means that $\mathrm{PH}$ assumption is violated. This problem would be clarified if the PH assumption were tested using the graphical approaches such as Kaplan-Meier curves and/or statistical methods such as the goodness-of-fit test and time-dependent covariates. The variants of the Cox model such as extended or stratified Cox regression model must be used when the aforementioned assumption is violated; otherwise, findings may be misleading ${ }^{2}$.

Second, the authors found that the SUA is not linearly associated with all-cause mortality. In this situation, typical regression models are not effective. The full-range associations can be examined more efficiently using the restricted cubic splines in both univariate and multivariate models ${ }^{3}$.

Finally, scientists usually combine the multiple specific outcomes such as cause-specific mortality into the 1 combined category such as all-cause mortality to improve the sample size and power for statistical test. Homogeneity of direct and strength of associations between the exposure of interest and multiple specific outcomes, as a vital assumption, must be established when a combined outcome is used in the study. This assumption is usually hard to establish; some advanced methods such as hierarchical regression models are introduced to effectively evaluate the association between exposure and multiple specific outcomes, even in low sample size studies. Hence, we suggest that Kang and colleagues use this new regression model in their present and future investigations ${ }^{4}$.

ERFAN AYUBI 10 , PhD, Department of Epidemiology, School of Public Health, Shahid Beheshti University of Medical Sciences, Tehran; SAEID SAFIRI (B), PhD, Managerial Epidemiology Research Center, Department of Public Health, School of Nursing and Midwifery, Maragheh University of Medical Sciences, Maragheh, and Department of Epidemiology and Biostatistics, School of Public Health, Tehran University of Medical Sciences, Tehran, Iran.

Address correspondence to Dr. S. Safiri, Assistant Professor of Epidemiology, Managerial Epidemiology Research Center, Maragheh University of Medical Sciences, Maragheh, Iran.

E-mail: saeidsafiri@gmail.com

\section{REFERENCES}

1. Kang E, Hwang SS, Kim DK, Oh KH, Joo KW, Kim YS, et al. Sex-specific relationship of serum uric acid with all-cause mortality in adults with normal kidney function: an observational study. J Rheumatol 2017;44:380-7.

2. Kleinbaum DG, Klein M. Survival analysis: a self-learning text. New York: Springer Science and Business Media; 2006.

3. Steyerberg E. Clinical prediction models: a practical approach to development, validation, and updating. New York: Springer Science and Business Media; 2008.

4. Richardson DB, Hamra GB, MacLehose RF, Cole SR, Chu H. Hierarchical regression for analyses of multiple outcomes. Am J Epidemiol 2015;182:459-67.

J Rheumatol 2018;45:8; doi:10.3899/jrheum.170425 